Revista de

Contabilidade e Organizações

www.rco.usp.br
DOI: http://dx.doi.org/10.11606/issn.1982-6486.rco.2019.158490
Journal of

Accounting and

Organizations

\title{
Efeitos da corrupção nas bolsas de valores na Crise Financeira de 2008
}

\author{
Effects of corruption on stock exchanges in the 2008 Financial Crisis
}

Ronaldo dos Santos Alves Rodrigues ${ }^{\mathrm{a}}$, Ademir Clemente ${ }^{\mathrm{a}}$

${ }^{a}$ Universidade Federal do Paraná

Palavras-chave

Corrupção.

Crise Financeira de 2008.

Corrupção Institucional.
Keywords

Corruption.

2008 Financial Crisis.

Institutional Corruption.
Informações do artigo

Recebido: 30 de maio de 2019

Aceito: 13 de novembro de 2019

Publicado: 28 de novembro de 2019

\section{Resumo}

O objetivo deste artigo é averiguar se os níveis diferenciados de corrupção se refletiram no comportamento das bolsas de valores ao redor do mundo na Crise Financeira de 2008. A Teoria da Escolha Institucional indica que se generalizaram práticas financeiras proporcionadoras de grandes ganhos, as quais escondiam elevado risco, no período anterior à Crise. Foram utilizadas informações da Transparency International e do Banco Mundial sobre a corrupção percebida e o nível de governança para uma amostra final de 56 (cinquenta e seis) países. Para esses países, foi identificada a principal bolsa de valores e, para cada uma, o principal índice de bolsa, cujas cotações diárias de final de pregão foram levantadas para o período de 2007 a 2009. Os principais resultados evidenciam que em países com maior corrupção percebida e menor governança a Crise Financeira de 2008 acarretou maior queda acumulada das bolsas de valores, seguida de quebra estrutural mais profunda, aumento da volatilidade e menor previsibilidade da queda. Conclui-se que, de modo geral, os efeitos da Crise Financeira de 2008 foram diferenciados conforme o nível de corrupção percebida e de governança, sendo mais graves nos países em que a corrupção se apresentava mais institucionalizada.

\begin{abstract}
The purpose of this paper is to determine if the different levels of corruption reflected in the behavior of stock exchanges around the World in the 2008 Financial Crisis. Institutional Choice Theory indicates that, in the period before the Crisis, financial practices that provided large gains and concealed high risk were widespread. We used information from the Transparency International and the World Bank on perceived corruption and the level of governance on a final sample of 56 (fifty-six) countries. For these countries, we identified the main stock exchange and, for each one, the main burse index, whose daily quoted prices we gathered for the period from 2007 to 2009. The main results showed that in countries with greater perceived corruption and lower governance, the 2008 Financial Crisis presented a larger accumulated decrease of the stock exchanges followed by deeper structural breakdown, increased volatility and lower predictability of the drop. We conclude that, in general, the effects of the 2008 Financial Crisis were different according to the level of perceived corruption and governance, being more serious in countries where corruption was more institutionalized.
\end{abstract}

\section{Implicações práticas}

Os resultados encontrados auxiliam as decisões de financiamento e de investimento em países tendo em conta o nível de corrupção percebida, bem como, a escolha quanto à compra ou venda de ativos em cenários de crise. Embora não ofereça métricas, provê uma indicação útil sobre o comportamento das bolsas em momentos de crise.

Copyright (C) 2019 FEA-RP/USP. Todos os direitos reservados

Autor correspondente: Tel. (41) 3360-4413

E-mail: ronaldo.sarodrigues@gmail.com (R.dos S. A. Rodrigues); ademirclemente@gmail.com (A. Clemente)

Universidade Federal do Paraná, Setor de Ciências Sociais Aplicadas. Av. Prefeito Lothário Meissner, 632 - Jardim Botânico, Curitiba/PR - 80210170 , Brasil. 


\section{INTRODUÇÃO}

As enormes quedas das bolsas de valores de 1929, 1987 e 2008 são fenômenos associados à falta de regulamentação, conjuntura adversa e expansão exagerada no período imediatamente anterior. Entretanto, a quebra de expectativas devido à perda de confiança na capacidade do mercado de manter ou recuperar o equilíbrio é comum a todos eles. Com a perda de confiança, os agentes se retraem e suspendem suas operações e a crise se aprofunda.

O mercado de ações, assim como o de títulos em geral, é formado por um conjunto de regras a respeito das trocas efetuadas sob o sistema de preços e é essencialmente diferenciado em relação aos demais. Esse mercado responde prontamente às percepções dos agentes, principalmente, analistas e investidores, e é um sinalizador primordial da conjuntura econômica, pois os seus preços são diretamente influenciados pelo nível de confiança.

A Transparency International divulga anualmente o Índice de Percepção da Corrupção (IPC) que indica o nível de corrupção percebida em 180 (cento e oitenta) países. O Banco Mundial divulga o Índice de Governança dos Países (do inglês WGI) em 200 (duzentos) países e representa o nível de governança dos países ao redor do mundo. Esses índices podem ser utilizados como termômetros para o nível de incerteza econômica, financeira e social dos países, vale dizer, representam o nível de confiança nas instituições. A confiança nas instituições é corroída pela corrupção. Quanto ao mercado, o efeito econômico da corrupção tem como premissa as suas imperfeições, tais como assimetria informacional e incerteza (Jain, Kuvet \& Pagano 2017).

Estudos anteriores evidenciaram a relação entre a corrupção e elementos institucionais, entendendo a corrupção como presente nas instituições e por elas determinadas (Glaser \& Saks, 2006; Dong \& Torgler, 2013; Dong, Dulleck \& Torgler, 2012; Lee \& Guven, 2013; Dzhumashev, 2014, Mironov, 2015; Smith, 2016; Ortiz-Ospima \& Roser, 2016; Salmon \& Serra, 2017), bem como a relação entre corrupção e crises financeiras (Kaufmann, 2009; Ivlevs \& Hinks, 2013; Gugiu \& Gugiu, 2016).

Segundo a Teoria da Escolha Institucional, as instituições são formadas por regras ou conjuntos de regras políticas, econômicas e culturais que intermediam a relação entre o indivíduo (agente) e a estrutura (sociedade) (Collier, 2002). Nessa perspectiva, o mundo externo constitui a estrutura institucional e influencia o mundo interior dos agentes. Ou seja, as escolhas dos agentes são limitadas e condicionadas pelas instituições. A corrupção, vista sob essa perspectiva, é uma escolha do agente e, na medida em que a corrupção está mais presente nas instituições, a escolha institucional da corrupção torna-se mais comum.

Em momentos de crise, ocorre perda de confiança nas instituições e os mercados tornam-se mais vulneráveis (Corsett, Pasenti \& Roubini, 1999; Morales \& Andreosso-O'callaghan, 2012). Em mercados em que a corrupção está mais institucionalizada, a perda de confiança torna-se maior, devido à ineficiência de mecanismos de combate e controle da corrupção, abuso do poder político para interesses privados, subornos, desvio de dinheiro, nepotismo, falta de eficácia do governo, baixa qualidade regulatória, entre outros.

Diante disso, propõe-se a seguinte questão de pesquisa: Os efeitos da Crise Financeira de 2008 sobre os mercados acionários ao redor do mundo foram diferenciados conforme o nível de corrupção dos países?

Busca-se contribuir para a literatura acerca da corrupção ao utilizar a Teoria da Escolha Institucional para analisá-la, visto que várias publicações acerca de corrupção não apresentam suporte teórico (Judge, McNatt \& Xu, 2011). Uma segunda contribuição reside em relacionar a corrupção ao mercado financeiro (Jain, Kuvvet \& Pagano, 2017; Paserman, 2017), ao admitir que os agentes do mercado financeiro percebem o contexto social em que estão inseridos e suas decisões são influenciadas pelo quadro institucional. Uma terceira contribuição se refere às respostas que os mercados dão aos eventos externos.

Contaminados pela Crise Financeira de 2008, os mercados mundiais apresentaram grandes quedas e se tornaram mais voláteis. Entretanto, os mercados foram afetados de forma diferenciada e aqui se busca relacionar os efeitos, em cada mercado, com a percepção da corrupção. Por fim, espera-se também contribuir para a literatura acerca das decisões de investimento em mercados com corrupção institucionalizada, visto que estudos anteriores evidenciaram que a corrupção influencia a alavancagem e disponibilidade de caixa das empresas (Smith, 2016). 


\section{ESCOLHA INSTITUCIONAL}

A Teoria da Escolha Institucional foi desenvolvida por Collier (2002), que adotou uma abordagem interdisciplinar das causas da corrupção. O modelo desenvolvido por Collier (2002) estabelece que a escolha individual é limitada pelas instituições sociais. Para o autor, as instituições são formadas por regras ou conjunto de regras políticas, econômicas e culturais que intermediam a relação entre o indivíduo (agente) e a estrutura (sociedade).

As regras tornam as pessoas socialmente ativas, formam a relação agente-estrutura e concedem à sociedade um padrão ou estrutura reconhecível. Qualquer mudança nas regras de uma sociedade redefine os agentes, instituições e as suas relações uns com os outros (Onuf, 1994 apud Collier, 2002). Ou seja, os agentes e a sociedade co-constituem um ao outro em um processo contínuo (Onuf, 1997 apud Collier, 2002). Segundo essa perspectiva teórica, o mundo é socialmente estruturado por regras sociais, ou seja, por instituições (Collier, 2002).

Judge, McNatt e Xu (2011) adaptaram o modelo desenvolvido por Collier (2002) e apontam a corrupção como uma escolha institucional. Segundo o modelo, o mundo externo constitui a estrutura institucional (regras) que influencia o mundo interno do agente na tomada de decisão. É no mundo externo que estão os recursos materiais que afetam os agentes quanto aos benefícios e custos esperados. Esse modelo é circular, o comportamento corrupto afeta a estrutura e as regras internalizadas dos agentes, representando um feedback. Nessa perspectiva, a corrupção é entendida como a obtenção de vantagens privadas legais ou ilegais, decorrente da utilização de poder, seja público ou privado (Collier, 2002; Judge, McNatt \& Xu, 2011; Ortiz-Ospina \& Roser, 2016). Para Judge, McNatt e Xu (2011), a corrupção ocorre nas organizações devido a comportamentos consolidados e compartilhados por seus membros. Na medida em que a corrupção se torna comum e generalizada, o custo esperado de ser punido se torna menor, devido à pouca possibilidade de detecção e punição (Dong, Dulleck \& Torgler, 2012).

A Crise Financeira de 2008 foi um acontecimento econômico imprevisto e de alcance mundial que, iniciado no mercado norte americano, foi rapidamente contagiando outros mercados e abalou a confiança nas organizações (Samarakoon, 2011; Morales \& Andreosso-O'callaghan, 2012; Kenourgios \& Dimitriou, 2015; Clemente, Juaniha \& Ribeiro, 2018). A origem da crise não está associada apenas ao aumento dos empréstimos a tomadores menos confiáveis - subprime - mas também à rede de conexões do mercado financeiro, dispersão do risco do sistema financeiro e perda de confiança na arquitetura financeira (Smick, 2009; Paulson Júnior, 2010; Shiller, 2012; Irwin, 2014).

O Quadro 1 apresenta os principais eventos divulgados para o mercado, concentrados no segundo semestre de 2008 (Bacen, 2008).

\begin{tabular}{cl}
\hline \multicolumn{1}{c}{ Data } & Evento \\
\hline $01 / 10 / 2008$ & Senado dos EUA aprova socorro financeiro de US\$ 700 bilhões \\
\hline $03 / 10 / 2008$ & Câmara dos EUA aprova pacote para conter a crise, valor chega a US\$ 700 bilhões \\
\hline $06 / 10 / 2008$ & Bolsas desabam no mundo todo; Bolsa de Moscou cai 19\% \\
\hline $07 / 10 / 2008$ & O pior da crise está por vir, segundo o FMI. É preciso restaurar a confiança no setor financeiro \\
\hline $13 / 10 / 2008$ & $\begin{array}{l}\text { Federal Reserve, Banco Central Europeu, Bank of England e Swiss National Bank anunciam medidas para } \\
\text { socorrer os bancos e aumentar a liquidez dos mercados }\end{array}$ \\
\hline $15 / 10 / 2008$ & $\begin{array}{l}\text { Pior pregão de Wall Street desde 1987 } \\
\text { Aumento dos empréstimos dos bancos dos EUA junto ao FED, média de US\$ 437bi }\end{array}$ \\
\hline & $\begin{array}{l}\text { Socorro financeiro do governo britânico por meio do Bank of England } \\
\text { Banco Central da Suíça ajuda o UBS com U\$\$ 5,2 bilhões e Credit Suisse levantou 10 bilhões de Francos } \\
\text { Suíços de fundos árabes } \\
\text { BCE aceita ativos de maior risco como garantia de empréstimos aos bancos }\end{array}$ \\
\hline Banco Central divulga regras para empréstimos em moeda estrangeira como garantia \\
$\begin{array}{l}\text { Devido a grandes perdas com derivativos, CVM exige detalhes, a partir do } 3^{\circ} \text { trimestre, sobre os instrumentos } \\
\text { financeiros utilizados }\end{array}$ \\
\hline Bolsas na Ásia fecham em forte queda, a de Tóquio cai 9,6\% \\
\hline $27 / 10 / 2008 / 2008$ & Bolsa de Hong Kong apresenta maior queda desde 1987 \\
\hline
\end{tabular}

Quadro 1. Eventos relacionados à Crise Financeira de 2008

Fonte: Elaborado pelos autores. 
No coração da Crise estava a perda de confiança na arquitetura financeira (Smick, 2009) e, em consequência, os mercados tornaram-se mais vulneráveis (Morales \& Andreosso-O'Callaghan, 2012). A solidez das instituições financeiras depende da confiança dos participantes do mercado, além de depender das relações econômicas (Smick, 2009; Paulson Junior, 2010).

Nessa situação, sob a ótica da Teoria da Escolha Institucional, os agentes do mercado financeiro incorporaram práticas que segundo Smick (2009, p. 292) eram de "'risco sem risco' com o benefício adicional de lucros enormes". Na medida em que essas práticas foram sendo adotadas para benefício privado sem a devida precaução quanto aos riscos, atitudes corruptas dos agentes se configuraram como legitimadas. Os rendimentos obtidos eram cada vez mais elevados e os reguladores do mercado tornaram-se complacentes em relação aos riscos adotados pelos agentes (Paulson Junior, 2010).

Segundo a Transparency International (2012) a crise na Grécia esteve relacionada com a corrupção, o que custou cerca de $€ 554$ milhões em 2011. Gugiu e Gugiu (2016) encontraram relação positiva entre a crise econômica da União Europeia e a percepção de corrupção. Kaufmann (2009) aponta que das diversas causas da crise financeira, a corrupção é uma das causas mais importantes. Ivlevs e Hinks (2013) encontraram relação entre os efeitos da Crise Financeira de 2008 com a corrupção em 30 economias em transição. Para os autores, a Crise contribuiu para o aumento no suborno, ou seja, indivíduos mais afetados pela Crise Financeira tinham maior probabilidade de se envolver em transações corruptas. Isso ocorre porque as vítimas da crise são alvos mais "fáceis" de suborno (Ivlevs \& Hinks, 2013).

Com base na literatura pesquisada, adota-se como hipótese geral que as bolsas de valores dos países com nível mais elevado de corrupção percebida foram mais afetadas pela Crise Financeira de 2008. No sentido de operacionalizar essa hipótese geral, enunciam-se as seguintes hipóteses:

$\mathbf{H}_{1}$ : Na Crise Financeira de 2008, os países em que a corrupção percebida era mais elevada apresentaram maior queda acumulada entre o pregão de maior queda e o seguinte.

$\mathbf{H}_{2}$ : A elevação da volatilidade das bolsas de valores causada pelo contágio da Crise Financeira de 2008 foi maior nos países com maior percepção de corrupção.

$\mathbf{H}_{3}$ : A previsibilidade da queda nas cotações das bolsas de valores na Crise Financeira de 2008 foi menor nos países com nível mais elevado de corrupção percebida.

$\mathbf{H}_{4}$ : A Crise Financeira de 2008 representou quebra estrutural de forma mais evidente nos países em que a percepção da corrupção era mais elevada.

$\mathbf{H}_{5}$ : A diferença entre as datas da maior queda e a da quebra estrutural foi maior nos países com menor índice de corrupção percebida.

\section{METODOLOGIA}

O universo deste estudo compreende os países para os quais se dispõe do IPC. Tal índice abrange 180 (cento e oitenta) países e é publicado pela Transparency International, sendo apresentado em uma escala de 0 a 100, em que 0 representa altamente corrupto e 100 isento de corrupção (Paldam, 2002; Transparency International, 2018). Segundo a Transparency International (2018), o IPC captura inúmeros aspectos da corrupção relativos a suborno, ausência de mecanismos de punição, leis adequadas sobre divulgação financeira, entre outros. O IPC apresenta-se vantajoso por estar disponível para um extenso período, pela quantidade de países pesquisados e também por refletir a percepção dos participantes do mercado financeiro (Paserman, 2017).

Adicionalmente, foi utilizado o índice de governança WGI, que compreende seis dimensões: Voz e Responsabilização, Estabilidade Política, Eficácia do Governo, Qualidade Regulatória, Estado de Direito, e Controle da Corrupção (Kaufmann, Kraay \& Mastruzzi, 2011). Um componente principal mostrou-se adequado para resumir a informação nelas contida.

Utilizamos IPC e WGI como proxies do nível de corrupção. Isso se assenta na ideia de que nos países que apresentam corrupção mais institucionalizada há maior percepção da corrupção e menor pontuação no indicador de governança. Nesse sentido, quanto menor o IPC e menor o WGI, mais institucionalizada a corrupção em certo país. 
Em seguida, foi identificada a principal bolsa de valores de cada país e o principal índice representativo do mercado. Como resultado, pôde-se associar a cada país um índice de bolsa de valores e levantar as cotações de fechamento de pregão para o período compreendido entre janeiro de 2007 e dezembro de 2009 (Righi \& Cereta, 2013; Paserman, 2017). Para as datas em que não houve negociação foi utilizada a cotação de fechamento do pregão anterior. Calculamos o retorno diário pela razão entre a variação entre cotações sucessivas e a cotação inicial.

A escolha do período se justifica pelo fato de a Crise Financeira de 2008 ter tido início no mercado norte americano em 2007 e perdurado até aproximadamente março de 2009 (Samarakoon, 2011; Kobayashi, Nakajima \& Inaba, 2012; Boubarker, Jouini \& Lahiani, 2016). Além disso, tomar como referência o mercado norte americano se justifica por ter sido o epicentro da Crise que contagiou os mercados financeiros ao redor do mundo (FfrenchDavis, 2010; Luchtenberg \& Vu, 2015; Boubaker, Jouini \& Lahiani, 2016).

\subsection{Hipóteses e procedimentos metodológicos}

Para as hipóteses $\mathrm{H}_{2}$ e $\mathrm{H}_{3}$ foi realizada análise a partir de janelas de 60 pregões antes e depois da maior queda (MacKinlay, 1997; Campbell, Lo \& MacKinlay, 1997).

Para as hipóteses $\mathrm{H}_{4}$ e $\mathrm{H}_{5}$, a janela utilizada foi o período pesquisado de 01/01/2007 a 31/12/2009 para verificar a existência de mudança estrutural (Gujarati, \& Porter, 2011; Shikida, Paiva \& Araújo Júnior, 2016). Utilizaram-se os Testes de Chow e Quandt ou sup-Wald. O Quadro 2 apresenta a definição das variáveis.

\begin{tabular}{|c|c|c|c|}
\hline Variáveis & Definição & Símbolo & Operacionalização \\
\hline Corrupção & $\begin{array}{l}\text { Índice de Percepção da } \\
\text { Corrupção }\end{array}$ & IPC & Índice da Transparency International \\
\hline Governança Mundial & $\begin{array}{l}\text { Índice de Governança } \\
\text { dos Países }\end{array}$ & WGI & Índice do Banco Mundial \\
\hline Maior queda & $\begin{array}{l}\text { Retorno negativo mais } \\
\text { acentuado }\end{array}$ & Queda & $R_{i t}=\operatorname{In}\left(P_{t} / P_{t-1}\right)$ \\
\hline Queda Acumulada & $\begin{array}{l}\text { Retorno negativo mais } \\
\text { acentuado acumulado } \\
\text { com o do pregão seguinte }\end{array}$ & Q_acum & $R_{i t}=\operatorname{In}\left(P_{t-1} / P_{t+1}\right)$ \\
\hline \multirow[t]{2}{*}{ Volatilidade } & \multirow{2}{*}{$\begin{array}{l}\text { Volatilidade na janela } \\
\text { de } 60 \text { pregões antes da } \\
\text { maior queda e } 60 \text { após }\end{array}$} & DIF_DP; RAZ_DP & $\begin{array}{l}\text { Diferença e Razão do desvio Padrão } \\
\text { nas janelas de estimação e de } \\
\text { comparação }\end{array}$ \\
\hline & & DIF_VAR; RAZ_VAR & $\begin{array}{l}\text { Diferença e Razão da variância nas } \\
\text { janelas de estimação e de comparação }\end{array}$ \\
\hline $\begin{array}{l}\text { Diferença entre a } \\
\text { previsão de queda e a } \\
\text { queda efetiva }\end{array}$ & $\begin{array}{l}\text { Diferença entre o retorno } \\
\text { predito e o retorno } \\
\text { calculado para o dia da } \\
\text { maior queda }\end{array}$ & Dif & $\begin{array}{l}\left(\text { Yestimado }_{i t}\right)=a+B_{i t} \\
\text { Dif }=\left(\text { Yestimado }_{i t}\right)-Y_{i t}\end{array}$ \\
\hline Quebra estrutural & $\begin{array}{l}\text { Mudança no nível, } \\
\text { dispersão, inclinação da } \\
\text { série temporal }\end{array}$ & Qe & $\begin{array}{l}\text { Estatística F do Teste de Quandt e } \\
\text { Teste de Chow }\end{array}$ \\
\hline Previsão da queda & $\begin{array}{l}\text { Diferença entre a data } \\
\text { do pregão de maior } \\
\text { queda e a data da quebra } \\
\text { estrutural }\end{array}$ & Dif_datas & $\begin{array}{l}\text { Correlação entre a diferença da data } \\
\text { do pregão de maior queda e data da } \\
\text { quebra estrutural }\end{array}$ \\
\hline
\end{tabular}

Quadro 2. Definição das variáveis

Fonte: Elaborado pelos autores.

As hipóteses formuladas, as respectivas variáveis e testes estão no Quadro 3. 


\begin{tabular}{cccc}
\hline Hipótese & Variáveis & Teste Estatístico & Sinal Esperado \\
\hline $\mathrm{H}_{1}$ & Q_acum; IPC; WGI & Correlação & $(+)$ \\
$\mathrm{H}_{2}$ & DIF_N; DIF_N1; DIF_DP; RAZ_DP; & Correlação & $(-)$ \\
$\mathrm{H}_{3}$ & DIF_VAR; RAZ_VAR; IPC; WGI & Correlação & $(-)$ \\
$\mathrm{H}_{4}$ & Dif; IPC; WGI & Correlação & $(+)$ \\
$\mathrm{H}_{5}$ & Qe; IPC; WGI & Correlação & $(+)$ \\
\hline
\end{tabular}

Quadro 3. Hipóteses, variáveis e testes estatísticos Fonte: Elaborado pelos autores.

\subsection{Amostra}

Para cada país foi identificada a principal bolsa de valores e buscado o seu principal índice. Em seguida, foram coletados os valores de fechamento diário de cada índice. Isso possibilitou o cálculo do retorno diário. Foram selecionados os países que apresentaram a maior queda no terceiro quadrimestre de 2008 , totalizando 56 , conforme o Quadro 4.

\begin{tabular}{lc}
\hline \multicolumn{1}{c}{ Países } & Quantidade \\
\hline Países listados no IPC em 2008 & 180 \\
Com dados faltantes & $(98)$ \\
Maior queda não ocorreu no terceiro quadrimestre de 2008 & $(26)$ \\
Amostra & 56 \\
\hline
\end{tabular}

Quadro 4. Amostra

Fonte: Elaborado pelos autores

\section{RESULTADOS}

Conforme mostrado pela Tabela 1, entre os países selecionados na amostra, a primeira grande queda devida à Crise ocorreu em 15/09/2008, na Ucrânia, e a última, no dia 01/12/2008, no Canadá. Do total da amostra, 21,42\% das quedas estão concentradas no dia 06/10/2008. Conforme o Quadro 1, essa data foi de grande volatilidade e queda acentuada em vários países.

A maior queda registrada em toda a amostra ocorreu na Rússia, no mesmo pregão de $06 / 10 / 2008$, igual a $20,7 \%$, e a menor queda foi observada em Malta, no dia 18/09/2008, com variação negativa de 4\%. Os Estados Unidos, que foram o epicentro da Crise Financeira de 2008, tiveram queda de 8\% no dia 15/10/2008. Na mesma data, no Brasil houve queda de 12\%, na Hungria 13\% e na Argentina, também 13\%. Esse pregão de 15/10/2008 foi reconhecido como o pior em Wall Street desde 1987 (Quadro 1).

A maior volatilidade em torno da maior queda também foi observada na Rússia, enquanto a menor volatilidade ocorreu em Malta. Assim, tem-se que o mercado que apresentou a maior queda também apresentou a maior volatilidade e que o mercado de menor queda foi também o de menor volatilidade em seu entorno.

Por meio da Análise de Componentes Principais (PCA), extrai-se um componente representando 85,83\% da variância observada nas variáveis que compõem o WGI. O valor de Kaiser-Meyer-Olkin (KMO) alcançou 0,917 , indicando alto nível de adequação da PCA. 
Tabela 1. Variáveis da pesquisa e estatísticas descritivas

\begin{tabular}{|c|c|c|c|c|c|c|c|c|}
\hline País & Índice & $\begin{array}{l}\text { Data } \\
\text { (2008) }\end{array}$ & IPC & WGI & $\begin{array}{c}\text { Queda } \\
(\%)\end{array}$ & $\underset{(\%)^{*}}{\text { Q_acum }^{*}}$ & $\begin{array}{c}\text { Vol_DP } \\
-60 /+60 * *\end{array}$ & $\begin{array}{l}\text { Vol_VAR } \\
-60 /+60 * *\end{array}$ \\
\hline Ucrânia & UAX & $15 / \mathrm{set}$ & 2,5 & $-0,5$ & 13,2 & 24,5 &, $0289 /, 0481$ & $0001 /, 0023$ \\
\hline Malta & MSE & $18 /$ set & 5,8 & 1,39 & 3,5 & 1,8 &, $0081 /, 0075$ & ,0001/,0001 \\
\hline Luxemburgo & LUXX & $29 /$ set & 8,3 & 1,85 & 11,2 & 7,9 & ,0214/,0370 &, $0005 /, 0014$ \\
\hline Irlanda & ISEQ & $29 /$ set & 7,7 & 1,74 & 14 & 6,4 &, $0355 /, 0392$ & ,0001/,0015 \\
\hline Bélgica & BFX & $29 / \mathrm{set}$ & 7,3 & 1,38 & 8,3 & 5,2 & ,0233/,0345 &, $0001 /, 0012$ \\
\hline Dinamarca & OMXN40 & 06/out & 9,3 & 2,05 & 8,7 & 9,7 & ,0231/,0392 & ,0001/,0015 \\
\hline Suécia & OMXS30 & 06/out & 9,3 & 1,92 & 7,5 & 9,8 & ,0234/,0381 &, $0006 /, 0014$ \\
\hline Finlândia & OMXH25 & 06/out & 9 & 1,99 & 8,9 & 12,1 & ,0234/,0344 &, $0006 /, 0012$ \\
\hline Países Baixos & AEX & 06/out & 8,9 & 1,79 & 9,6 & 10,6 &, $0240 /, 0414$ & ,0006/,0017 \\
\hline França & FCHI & 06/out & 6,9 & 1,41 & 9,5 & 8,9 & ,0217/,0396 & ,0005/,0016 \\
\hline Chile & SPCLXIPSA & 06/out & 6,9 & 1,27 & 6,2 & 10,6 & ,0158/,0259 & ,0003/,0007 \\
\hline Portugal & PSI20 & 06/out & 6,1 & 1,18 & 10,4 & 11,6 & ,0194/,0289 & ,0004/,0008 \\
\hline África do Sul & JTOPI & 06/out & 4,9 & 0,35 & 8 & 5,1 & ,0239/,0362 &, $0006 /, 0013$ \\
\hline Itália & FTMIB & 06/out & 4,8 & 0,64 & 8,6 & 9,2 & ,0200/,0384 & ,0004/,0015 \\
\hline Lituânia & OMXVGI & 06/out & 4,6 & 0,76 & 9,1 & 17,4 & ,0156/,0331 &, $0002 /, 0011$ \\
\hline Peru & XU100 & 06/out & 4,6 & 0,01 & 9 & 9,1 &, $0280 /, 0350$ & ,0008/,0012 \\
\hline Rússia & IMOEX & 06/out & 2,1 & $-0,8$ & 20,7 & 21,6 &, $0517 /, 0655$ & ,0037/,0043 \\
\hline Jordânia & AMGNRLX & $07 /$ out & 5,1 & 0,07 & 4,4 & 8,3 & ,0155/,0242 &, $0002 /, 0006$ \\
\hline Letônia & OMXRGI & 07/out & 5 & 0,67 & 7,9 & 14,1 & ,0168/,0310 & ,0003/,0010 \\
\hline Sérvia & BELEX15 & 07/out & 3,4 & $-0,28$ & 10,9 & 20,5 & ,0186/,0336 &, $0004 /, 0011$ \\
\hline Estônia & OMXTGI & 08/out & 6,6 & 1,19 & 7 & 1,3 & ,0189/,0264 & ,0004/,0007 \\
\hline Indonésia & JKSE & 08/out & 2,6 & $-0,51$ & 11 & 10,3 & ,0224/,0326 &, $0005 /, 0011$ \\
\hline Cazaquistão & KASE & 08/out & 2,2 & $-0,58$ & 12,9 & 6,6 & ,0231/,0582 & ,0005/,0034 \\
\hline Suíça & SSMI & 10/out & 9 & 1,89 & 8,1 & $-2,7$ & ,0209/,0318 & ,0004/,0010 \\
\hline Austrália & AXJO & 10/out & 8,7 & 1,79 & 8,7 & 3,3 &, $0204 /, 0280$ & ,0004/,0008 \\
\hline Reino Unido & FTSE & 10/out & 7,7 & 1,61 & 9,3 & 1,3 & ,0240/,0338 &, $0006 /, 0011$ \\
\hline Espanha & IBEX & 10/out & 6,5 & 1,01 & 9,6 & $-0,5$ &, $0244 /, 0353$ & ,0006/,0012 \\
\hline Rep. Checa & PX & 10/out & 5,2 & 1 & 16,2 & 6,2 & $0267 /, 0465$ & ,0007/,0022 \\
\hline Polônia & WIG & 10/out & 4,6 & 0,75 & 8,3 & 6,6 &, $0185 /, 0274$ &, $0003 /, 0008$ \\
\hline Croácia & CRBEX & 10/out & 4,4 & 0,39 & 10,8 & -4 & ,0211/,0399 & ,0004/,0016 \\
\hline Colômbia & COLCAP & 10/out & 3,8 & $-0,39$ & 8,9 & 0,2 &, $0143 /, 0258$ &, $0002 /, 0007$ \\
\hline Peru & SPBLPGPT & 10/out & 3,6 & $-0,29$ & 11,4 & $-1,4$ & ,0277/,0423 & ,0008/,0018 \\
\hline Estados Unidos & DJI & $15 /$ out & 7,7 & 1,48 & 8,2 & 3,6 & ,0281/,0344 & ,0008/,0012 \\
\hline Hungria & BUX & 15/out & 5,1 & 0,91 & 12,6 & 21,6 &, $0285 /, 0410$ & ,0008/,0017 \\
\hline Brasil & BVSP & 15/out & 3,5 & $-0,01$ & 12,1 & 13,2 & ,0398/,0445 & ,0016/,0020 \\
\hline Argentina & MERV & 15/out & 2,9 & $-0,33$ & 13 & 12,9 & $0327 /, 0417$ & ,0011/,0017 \\
\hline Nova Zelândia & NZ50 & 16/out & 9,3 & 1,9 & 4,9 & 3,4 &, $0166 /, 0143$ & ,0003/,0002 \\
\hline Japão & $\mathrm{N} 225 \mathrm{E}$ & 16/out & 7,3 & 1,31 & 12,1 & 9,4 & ,0310/,0406 & ,0010/,0017 \\
\hline México & MXX & 22/out & 3,6 & $-0,18$ & 7,3 & 12,7 & ,0274/,0311 & ,0008/,0010 \\
\hline Cingapura & FTSTIL & 24/out & 9,2 & 1,71 & 8,7 & 4,6 &, $0264 /, 0253$ & ,0007/,0006 \\
\hline Coreia do Sul & KS11 & 24/out & 5,5 & 0,78 & 8,7 & 5,9 & ,0096/,0107 & ,0007/,0001 \\
\hline Grécia & ATG & 24/out & 4,7 & 0,67 & 10,2 & 6,3 &, $0281 /, 0290$ &, $0008 /, 0008$ \\
\hline Guatemala & BSESN & 24/out & 3,1 & $-0,62$ & 11,6 & 13,8 & ,0294/,0329 & ,0009/,0011 \\
\hline Hong Kong & HSI & 27/out & 8,1 & 1,63 & 13,6 & 0,2 & ,0349/,0384 & ,0012/,0015 \\
\hline
\end{tabular}

Fonte: Elaborada pelos autores.

Nota: $N^{*}$ Sinal negativo indica recuperação com variação positiva acumulada. ** Volatilidades medidas como Desvio Padrão dos retornos, 60 pregões antes e 60 pregões depois da maior queda. 
Tabela 1. Variáveis da pesquisa e estatísticas descritivas (continuação)

\begin{tabular}{rcccccccc}
\hline País & Índice & $\begin{array}{c}\text { Data } \\
\mathbf{( 2 0 0 8 )}\end{array}$ & IPC & WGI & $\begin{array}{c}\text { Queda } \\
\mathbf{( \% )}\end{array}$ & $\begin{array}{c}\text { Q_acum } \\
\mathbf{( \% ) * *}\end{array}$ & $\begin{array}{c}\text { Vol_DP } \\
\mathbf{- 6 0 / + 6 0 * *}\end{array}$ & $\begin{array}{c}\text { Vol_VAR } \\
\mathbf{- 6 0 / + 6 0 * * *}\end{array}$ \\
\hline Áustria & ATX & $27 /$ out & 8,1 & 1,81 & 10,3 & 6,8 &, $0402 /, 0383$ &, $0016 /, 0015$ \\
Catar & QSI & $27 /$ out & 6,5 & 0,55 & 9,4 & 10,8 &, $0355 /, 0319$ &, $0013 /, 0010$ \\
Tailândia & SETI & $27 /$ out & 3,5 & $-0,31$ & 11,1 & 8,4 &, $0274 /, 0243$ &, $0008 /, 0006$ \\
Líbano & BLSI & $27 /$ out & 3 & $-0,77$ & 6,8 & 2,3 &, $0158 /, 0131$ &, $0003 /, 0002$ \\
Filipinas & PSI & $27 /$ out & 2,3 & $-0,56$ & 13,1 & 13,6 &, $0251 /, 0223$ &, $0006 /, 0005$ \\
Nigéria & NGSEINDEX & $28 /$ out & 2,7 & $-1,12$ & 3,6 & 6,9 &, $0113 /, 0179$ &, $0001 /, 0003$ \\
Noruega & OSEBX & $06 /$ nov & 7,9 & 1,82 & 10,5 & 7,6 &, $0458 /, 0377$ &, $0021 /, 0014$ \\
Bulgária & SOFIX & $18 /$ nov & 3,6 & 0,21 & 11,4 & 7 &, $0340 /, 0217$ &, $0012 /, 0005$ \\
Eslovênia & SBITOP & $20 /$ nov & 6,7 & 1,12 & 8,4 & 7,1 &, $0308 /, 0174$ &, $0010 /, 0003$ \\
Maurício & MDEX & $21 /$ nov & 5,5 & 0,88 & 6,4 & 12,4 &, $0189 /, 0140$ &, $0004 /, 0002$ \\
Israel & TA35 & $24 /$ nov & 6 & 0,73 & 6,8 & 1,6 &, $0275 /, 0213$ &, $0008 /, 0005$ \\
Canadá & GSPTSE & $01 /$ dez & 8,7 & 1,79 & 9,8 & 10,7 &, $0416 /, 0237$ &, $0017 /, 0006$ \\
\hline
\end{tabular}

Fonte: Elaborada pelos autores.

Nota: N* Sinal negativo indica recuperação com variação positiva acumulada. ** Volatilidades medidas como Desvio Padrão dos retornos, 60 pregões antes e 60 pregões depois da maior queda.

A maior queda registrada em toda a amostra ocorreu na Rússia, no mesmo pregão de 06/10/2008, igual a 20,7\%, e a menor queda foi observada em Malta, no dia 18/09/2008, com variação negativa de 4\%. Os Estados Unidos, que foram o epicentro da Crise Financeira de 2008, tiveram queda de 8\% no dia 15/10/2008. Na mesma data, no Brasil houve queda de 12\%, na Hungria 13\% e na Argentina, também 13\%. Esse pregão de 15/10/2008 foi reconhecido como o pior em Wall Street desde 1987 (Quadro 1).

A maior volatilidade em torno da maior queda também foi observada na Rússia, enquanto a menor volatilidade ocorreu em Malta. Assim, tem-se que o mercado que apresentou a maior queda também apresentou a maior volatilidade e que o mercado de menor queda foi também o de menor volatilidade em seu entorno.

Por meio da Análise de Componentes Principais (PCA), extrai-se um componente representando 85,83\% da variância observada nas variáveis que compõem o WGI. O valor de Kaiser-Meyer-Olkin (KMO) alcançou 0,917, indicando alto nível de adequação da PCA.

\subsection{Testes estatísticos}

Conforme a Tabela 2, o Coeficiente de Correlação de Pearson entre a queda acumulada no pregão de maior queda e no seguinte com o IPC é significativa a 1\%, enquanto a correlação com o WGI, a 5\%.

Tabela 2. $\mathrm{H}_{1}$ : Correlação entre queda acumulada, IPC e WGI

\begin{tabular}{lrcrc}
\hline & \multicolumn{2}{c}{ IPC } & \multicolumn{2}{c}{ WGI } \\
\cline { 2 - 5 } & $\mathrm{r}$ & $\mathrm{t}$ & $\mathrm{r}$ & $\mathrm{t}$ \\
\hline Q_acum & 0,32884 & $2,55877^{* * * *}$ & 0,28216 & $2,16126^{* *}$ \\
\hline Fonte: Elaborada pelos autores. & & &
\end{tabular}

Em relação ao IPC, tem-se forte evidência favorável a $\mathrm{H}_{1}$. Quanto ao WGI, a evidência não é tão clara, mas o coeficiente se apresenta com o sinal esperado e é significativo a $5 \%$.

Uma das dimensões que o IPC captura é o combate e controle à corrupção. Países com baixos mecanismos de punição facilitam a corrupção e em sociedades com mecanismos de punição mais eficientes os agentes são mais receosos e, se agirem, terão maiores cuidados (Paldam, 2002; Salmon \& Serra, 2017). Quando não existem controle e punição, mesmo que de natureza moral, a corrupção torna-se atrativa e funciona como um negócio (Paldam, 2002) devido ao baixo risco e às possibilidades de ganhos. 
$\mathrm{O}$ teste de $\mathrm{H}_{2}$ envolve a comparação da volatilidade no período anterior à maior queda com a volatilidade no período posterior, utilizando janelas de 60 (sessenta) pregões. As variáveis utilizadas para medir o acréscimo de volatilidade e os respectivos resultados são apresentados na Tabela 3.

Tabela 3. $\mathrm{H}_{2}$ : Correlação e estatística $t$ de Student para o aumento de volatilidade

\begin{tabular}{ccccc}
\hline & \multicolumn{2}{c}{ IPC } & \multicolumn{2}{c}{ WGI } \\
\cline { 2 - 5 } & $\mathrm{r}$ & $\mathrm{t}$ & $\mathrm{r}$ & $\mathrm{t}$ \\
\hline DIF_DP & $-0,1559$ & $-1,1705$ & $-0,1158$ & $-0,8644$ \\
\hline RAZ_DP & $-0,1329$ & $-0,9948$ & $-0,0964$ & $-0,7184$ \\
\hline DIF_VAR & $-0,2216$ & $-1,6853^{* *}$ & $-0,1786$ & $-1,3465$ \\
\hline RAZ_VAR & $-0,1585$ & $-1,1904$ & $-0,1146$ & $-0,8556$ \\
\hline
\end{tabular}

Fonte: Elaborada pelos autores.

Nota: DIF_DP - diferença entre desvios padrões, RAZ_DP - razão entre desvios padrões, DIF_ VAR - diferença entre variâncias, RAZ_VAR - razão entre variâncias.

Muito embora todos os coeficientes calculados apresentem o sinal esperado, apenas um deles é significativo ao nível de 5\%. Diante desses resultados, é interessante observar a relação envolvendo a diferença entre variâncias e a maior queda nas bolsas pesquisadas.

O diagrama de dispersão do Gráfico 1 mostra um padrão claramente decrescente. O coeficiente de correlação entre o aumento da volatilidade, medida pela diferença entre a variância nos 60 pregões anteriores e nos 60 pregões imediatamente posteriores à maior queda com a maior queda, igual a $-0,38$, é significativo a $1 \%$.

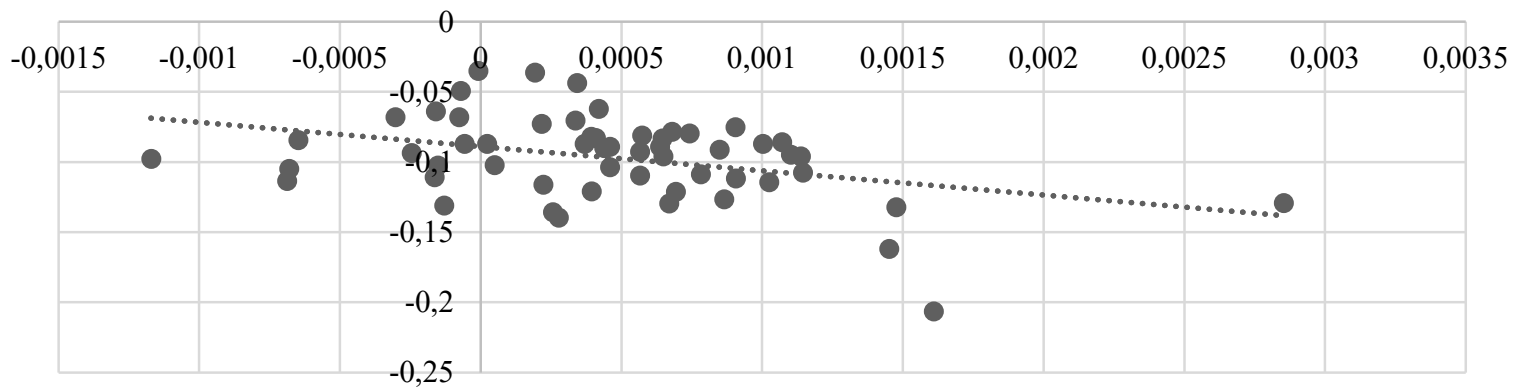

Gráfico 1. Relação entre aumento da volatilidade e queda dos retornos Fonte: Elaborado pelos autores.

Nos mercados com menor percepção da corrupção e baixa governança os índices de desenvolvimento são mais elevados (Amavilah, 2009; Marino, Soares, Luca \& Vasconcelos, 2016; Ortiz-Ospima \& Roser, 2016; Vale, 2018), os investidores percebem maior estabilidade e se sentem menos sujeitos a incertezas. Os países com maior percepção de corrupção, em geral, apresentam nível de desenvolvimento mais baixo, mercados menos desenvolvidos e maior instabilidade.

Como mostra o Gráfico 1, nos países que apresentaram maior queda houve maior aumento da volatilidade. Nesses mercados, os investidores reagem reduzindo drasticamente suas expectativas e causando grandes oscilações, enquanto nos mercados mais desenvolvidos, os investidores tendem a manter suas expectativas, apostando na recuperação, e a não reagir de forma tão acentuada.

Ito e Hashimoto (2005) apontam que uma crise pode contagiar os mercados devido ao comportamento dos investidores. Os investidores compartilham informações ou fragmentos de informações que funcionam com efeito cascata e fazem os demais investidores revisarem suas expectativas. Dessa forma, na Crise Financeira de 2008, o aumento da volatilidade está correlacionado com o tamanho da queda nos mercados financeiros e essa queda está correlacionada com a percepção de corrupção; todavia, não se colhe evidência de que o aumento da volatilidade esteja diretamente correlacionado com a percepção da corrupção.

Provavelmente, a relação entre aumento da volatilidade e percepção de corrupção recebe várias influências, tais como o tamanho do mercado, seu nível de concentração, participação de capitais estrangeiros, além de fatores culturais e econômicos (Glaser \& Saks, 2006; Dong \& Torgler, 2013; Dong, Dulleck \& Torgler, 2012; Dzhumashev, 2014; Salmon \& Serra, 2017). Dessa forma, $\mathrm{H}_{2}$ resulta estatisticamente não evidenciada, embora isso possa ser atribuído a limitações da amostra. 
A Tabela 4 mostra os resultados dos testes estatísticos relativos a $\mathrm{H}_{3}$.

Tabela 4. $\mathrm{H}_{3}$ : Correlação e t de Student para previsibilidade

\begin{tabular}{lcccc}
\hline & \multicolumn{2}{c}{ IPC } & \multicolumn{2}{c}{ WGI } \\
\cline { 2 - 5 } & $\mathrm{r}$ & $\mathrm{t}$ & $\mathrm{r}$ & $\mathrm{t}$ \\
\hline ABS_DIF & $-0,31981$ & $-2,50326^{* * *}$ & $-0,27583$ & $-2,12819^{* *}$ \\
\hline Fonte: Elaborada pelos autores. \\
$\begin{array}{l}\text { Nota: ABS_DIF -Diferença absoluta entre o retorno predito e o retorno calculado para o dia } \\
\text { da maior queda }\end{array}$
\end{tabular}

Os resultados mostram que a $\mathrm{H}_{3}$ encontra evidência na amostra. Os coeficientes estimados apresentam os sinais esperados e a correlação com o IPC é significativa ao nível de 1\%, e com o WGI, a 5\%. Os dados mostram que a previsibilidade da queda foi menor nos países com nível mais elevado de percepção da corrupção.

Informações advindas de mercados associados a nível de confiança mais baixo despertam maior incerteza, dificultando a previsibilidade e prejudicando a formação de expectativas (Ito \& Hashimoto 2005; Paserman, 2017). As notícias são menos confiáveis e podem ser irreais, podendo tanto aumentar quanto esconder a gravidade das situações relatadas, aumentado a incerteza dos investidores (Paserman, 2017). Essa incerteza ampliada reduz a informação efetivamente útil e torna menos previsível o comportamento dos investidores e do mercado.

Constata-se que houve quebra estrutural em todos os países da amostra. De fato, esses resultados indicam que a Crise Financeira de 2008 foi um evento externo que abalou todos os mercados de ações ao redor do mundo. Estimou-se a correlação entre os valores de F, uma vez ordenados, com o IPC e o WGI, obtendo-se os resultados da Tabela 5 .

Tabela 5. $\mathrm{H}_{4}$ : Correlação entre a ordem das estatísticas F, IPC e WGI

\begin{tabular}{lcccc}
\hline & \multicolumn{2}{c}{ IPC } & \multicolumn{2}{c}{ WGI } \\
\cline { 2 - 5 } & $\mathrm{r}$ & $\mathrm{t}$ & $\mathrm{r}$ & $\mathrm{t}$ \\
\hline F_Ord (Qe) & $-0,4332$ & $-3,5641^{* * *}$ & $-0,3859$ & $-3,1023^{* * *}$ \\
\hline Fonte: Elaborada pelos autores. & & &
\end{tabular}

Os valores encontrados foram todos significativos ao nível de 1\% e, assim, pode-se concluir que a quebra estrutural foi mais evidente nos países com maior percepção de corrupção. Dito de outra forma, os mercados que apresentam corrupção mais institucionalizada experimentaram quebra estrutural mais pronunciada.

A literatura aponta que a corrupção possui causas institucionais e que diferem entre os mercados (Dong \&Torgler, 2013; Lee \& Guven, 2013; Dzhumashev, 2014; Ortiz-Ospima \& Roser, 2016). Dito isso, os resultados encontrados apontam uma relação entre eventos externos, Crise Financeira de 2008, com características internas, corrupção. A ausência de mecanismos de controle da corrupção e de governança representam maior exposição aos eventos econômicos externos, tornando o mercado interno mais vulnerável.

Em relação a $\mathrm{H}_{5}$ os coeficientes de correlação estimados apresentam os sinais esperados, o relativo ao IPC $(0,2998)$ é significativo ao nível de $5 \%$, e o relativo ao WGI $(0,3413)$, a $1 \%$. Assim, colhe-se evidência de que os mercados acionários dos países com menor índice de corrupção percebida apresentaram um espaçamento maior entre a data da quebra estrutural e a data da maior queda. Os mercados que possuem a corrupção menos institucionalizada apresentaram maior resistência à mudança estrutural, como era esperado. Porém, contrariando o esperado, mercados tidos como sujeitos a larga margem de corrupção logo apresentaram reacomodação estrutural.

Devido a características internas, os mercados exibem mudanças em maior ou menor prazo. Os agentes, em mercados em que a corrupção é mais institucionalizada, possuem maior desconfiança e se sentem mais vulneráveis em situações de crise (Corsett, Pasenti \& Roubini, 1999; Smick, 2009; Paulson Jr, 2010; Morales \& Andreosso-O'callaghan, 2012), fazendo com que, ao perceberem mudanças, rapidamente as incorporem às novas regras (Collier, 2002). No entanto, nos mercados em que a corrupção é menos institucionalizada ocorre o inverso. Devido à maior confiança dos agentes, a reposta à mudança tende a ser mais demorada.

Considerados em conjunto, os testes de $\mathrm{H}_{4}$ e $\mathrm{H}_{5}$ indicam que a queda dos retornos nas bolsas de valores devida ao contágio foi seguida de uma quebra estrutural mais profunda e mais imediata nos países em que a corrupção se apresentava mais institucionalizada. Isso bem representa a relativa falta de confiança dos agentes na capacidade de recuperação dos mercados. 


\section{CONCLUSÃO}

A Crise Financeira de 2008, assim como a Crise de 1987 e de 1929, constituem eventos ímpares, marcantes e reveladores. São situações raras em que os mercados se desequilibram e mostram características que normalmente não são perceptíveis. Os mercados acionários, em particular, estão sujeitos a um conjunto de fatores reais e expectacionais que tornam complexa a sua análise e a sua previsão, mesmo em situações normais e, por isso, as crises representam imenso potencial de informação. Sem dúvida, a Crise Financeira de 2008, dada a sua profundidade e abrangência, é fonte valiosa de conhecimento sobre o comportamento das bolsas de valores.

Neste artigo, a hipótese geral de que o efeito da Crise Financeira de 2008 se fez mais pronunciado nas bolsas de valores dos países em que o nível da corrupção percebida era mais elevado, ou em que o nível de governança era mais baixo, resulta confirmada.

Segundo a Teoria da Escolha Institucional o mercado, enquanto instituição, precisa contar com elevado nível de confiança para o seu adequado funcionamento. Em situações normais, os agentes já se sentem menos seguros em mercados com elevada institucionalização da corrupção, pois a corrupção corrói a confiança nas instituições devido à busca de gratificação pessoal ou de um grupo, à margem da lei e dos princípios morais. Entretanto, isso se agrava e se potencializa a partir do contágio de uma crise de dimensões mundiais, como a Crise Financeira de 2008.

A pesquisa desenvolvida permite concluir que a Crise Financeira de 2008 realmente provocou efeitos diferenciados nas bolsas de valores ao redor do mundo e que um dos fatores a explicar esse fenômeno é o nível de corrupção percebida e de governança dos países.

Certamente, vários outros fatores intervêm na relação aqui analisada, como já apontado, indicando a necessidade de pesquisa adicional. Nesse sentido, devido ao método estatístico utilizado, o estudo apresenta apenas evidências da relação entre a corrupção e os efeitos Crise Financeira de 2008, sendo necessários métodos mais robustos para aprofundamento da análise. Apesar disso, pôde ser evidenciado o efeito diferenciado da Crise Financeira de 2008 conforme o nível de institucionalização da corrupção.

Assim como um organismo acometido por uma infecção tem todo o seu funcionamento modificado, as sociedades sujeitas a elevado nível de corrupção tentam impor algum controle por via de legislação e regulação que significam, na prática, dificuldades adicionais e crescentes aos negócios de boa-fé. Dessa forma, a corrupção, em vez de ser considerada um lubrificante para o mundo dos negócios segundo uma visão romântica e de curto prazo, constitui, com o passar do tempo, uma doença endêmica que inibe os negócios por falta de confiança e impõe pesadas restrições ao desenvolvimento econômico e social, principalmente em situação de crise.

A Crise Financeira de 2008 tornou evidente que a institucionalização da corrupção fragiliza pesadamente os mercados financeiros, tornando-os mais suscetíveis à queda das cotações e exacerbação da volatilidade, com maiores dificuldades de recuperação.

\section{REFERÊNCIAS}

Amavilah, V. H. (2009). Domestic Resources, Governance, Global Links, and the Economic Performance of SubSaharan Africa. Resource \& Engineering Economics Publications Services. DOI: http://dx.doi.org/10.2139/ ssrn.1359944.

Bacen (2008) Relatório de estabilidade financeira. Brasília - DF, 3(7), 140p.

Boubaker, S., Jouini, J., \& Lahiani, A. (2016). Financial contagion between the US and selected developed and emerging countries: The case of the subprime crisis. The Quarterly Review of Economics and Finance, 61, 14-28. DOI: https://doi.org/10.1016/j.qref.2015.11.001.

Campbell, J., Lo, A W., Mackinlay, A. (1997). The Econometric of Financial Markets. New Jersey: Princeton University Press.

Clemente, A., Juaniha, A. M., \& Ribeiro, F. (2017). Efeitos Da Crise Financeira De 2008 E Da Adoção Das IFRS Sobre O Matching Principle: Um Estudo Sobre As Empresas Industriais Listadas Na Bm\&Fbovespa (20052015). Contabilidade Vista \& Revista, 28(3), 133-157.

Corsetti, G., Pesenti, P., \& Roubini, N. (1999). What caused the Asian currency and financial crisis?. Japan and the world economy, 11(3), 305-373. DOI: https://doi.org/10.1016/S0922-1425(99)00019-5. 
Collier, M. W. (2002). Explaining corruption: An institutional choice approach. Crime, Law and Social Change, $38(1), 1-32$.

Dong, B., Dulleck, U., \& Torgler, B. (2012). Conditional corruption. Journal of Economic Psychology, 33(3), 609627. DOI: https://doi.org/10.1016/j.joep.2011.12.001.

Dong, B., \& Torgler, B. (2013). Causes of corruption: Evidence from China. China Economic Review, 26, 152169. DOI: https://doi.org/10.1016/j.chieco.2012.09.005.

Dzhumashev, R. (2014). Corruption and growth: The role of governance, public spending, and economic development. Economic Modelling, 37, 202-215. DOI: https://doi.org/10.1016/j.econmod.2013.11.007.

Ffrench-Davis, R. (2010). O Impacto da crise global na América Latina. Revista Tempo do Mundo - Instituto de Economia Aplicada (IPEA), 2 (89-104).

Glaeser, E. L., \& Saks, R. E. (2006). Corruption in America. Journal of public Economics, 90(6-7), 1053-1072. DOI: https://doi.org/10.3386/w10821.

Gugiu, M. R., \& Gugiu, P. C. (2016). Economic Crisis and Corruption in the European Union. Journal of Methods and Measurement in the Social Sciences, 7(1), 1-22. DOI: https://doi.org/10.2458/v7i1.19398.

Gujarati, D. N., \& Porter, D. C. (2011). Econometria Básica. Amgh Editora.

Irwin, N. (2014). Os grandes alquimistas financeiros: as histórias secretas dos presidentes dos três principais bancos centrais do mundo em uma economia em chamas. Rio de Janeiro: Elsevier.

Ito, T., \& Hashimoto, Y. (2005). High-frequency contagion of currency crises in Asia. Asian Economic Journal, 19(4), 357-381. DOI: https://doi.org/10.3386/w9376.

Ivlevs, A., \& Hinks, T. (2013). Global economic crisis and corruption experience: Evidence from transition economies. Economics Working Paper Series, University of the West of England.

Jain, P. K., Kuvvet, E., \& Pagano, M. S. (2017). Corruption's impact on foreign portfolio investment. International Business Review, 26(1), 23-35. DOI: https://doi.org/10.1016/j.ibusrev.2016.05.004.

Judge, W. Q., McNatt, D. B., \& Xu, W. (2011). The antecedents and effects of national corruption: A meta-analysis. Journal of World Business, 46(1), 93-103. DOI: https://doi.org/10.1016/j.jwb.2010.05.021.

Kaufmann, D. (2009). Corruption and the Global Financial Crisis. Disponível em: https://www.brookings.edu/ opinions/corruption-and-the-global-financial-crisis/. Acesso em outubro/2019.

Kaufmann, D., Kraay, A., \& Mastruzzi, M. (2011). The worldwide governance indicators: methodology and analytical issues. Hague Journal on the Rule of Law, 3(2), 220-246. DOI: https://oi.org/10.1017/ S1876404511200046.

Kenourgios, D., \& Dimitriou, D. (2015). Contagion of the Global Financial Crisis and the real economy: A regional analysis. Economic Modelling, 44, 283-293. DOI: https://doi.org/10.1016/j.econmod.2014.10.048.

Kobayashi, K., Nakajima, T., \& Inaba, M. (2012). Collateral constraint and news-driven cycles. Macroeconomic Dynamics, 16(5), 752-776. DOI: https://doi.org/10.1017/S1365100510000829.

Lee, W. S., \& Guven, C. (2013). Engaging in corruption: The influence of cultural values and contagion effects at the microlevel. Journal of Economic Psychology, 39, 287-300. DOI: https://doi.org/10.1016/j.joep.2013.09.006.

Luchtenberg, K. F., \& Vu, Q. V. (2015). The 2008 financial crisis: Stock market contagion and its determinants. Research in International Business and Finance, 33, 178-203. DOI: https://doi.org/10.1016/j.ribaf.2014.09.007

Mackinlay, A. (1997). Event Studies in Economic and Finance. Journal of Economic Literature, 35, p. 13-39.

Marino, P. D. B. L. P., Soares, R. A., De Luca, M. M. M., \& de Vasconcelos, A. C. (2016). Indicadores de governança mundial e sua relação com os indicadores socioeconômicos dos países do Brics. Revista de Administração Pública, 50(5), 721-744. DOI: http://dx.doi.org/10.1590/0034-7612144359.

Morales, L., \& Andreosso-O'Callaghan, B. (2012). The current global financial crisis: Do Asian stock markets show contagion or interdependence effects?. Journal of Asian Economics, 23(6), 616-626. DOI: https://doi. org/10.1016/j.asieco.2012.09.002.

Mironov, M. (2015). Should one hire a corrupt CEO in a corrupt country?. Journal of Financial Economics, 117(1), 29-42. DOI: https://doi.org/10.1016/j.jfineco.2014.03.002. 
Ortiz-Ospina, E, Roser, M. (2016). Corruption. Disponível em: https://ourworldindata.org/corruption. Acesso: Abril 2019.

Paldam, M. (2002). The cross-country pattern of corruption: economics, culture and the seesaw dynamics. European Journal of Political Economy, 18(2), 215-240. DOI: https://doi.org/10.1016/S0176-2680(02)00078-2.

Paulson Jr, H. M. (2010). A beira do abismo financeiro: a corrida para salvar a economia global do colapso. São Paulo: Campus.

Paserman, M. (2017). Comovement or safe haven? The effect of corruption on the market risk of sovereign bonds of emerging economies during financial crises. Journal of International Money and Finance, 76, $106-132$. DOI: https://doi.org/10.1016/j.jimonfin.2017.06.001.

Righi, B. M., \& Ceretta, P. S. (2013). Efeito da crise de 2007/2008 na transmissão internacional de volatilidade no mercado de capitais brasileiro. REAd-Revista Eletrônica de Administração, 19(2). DOI: http://dx.doi. org/10.1590/S1413-23112013000200005.

Samarakoon, L. P. (2011). Stock market interdependence, contagion, and the US financial crisis: The case of emerging and frontier markets. Journal of International Financial Markets, Institutions and Money, 21(5), 724-742.

Salmon, T. C., \& Serra, D. (2017). Corruption, social judgment and culture: An experiment. Journal of Economic Behavior \& Organization, 142, 64-78. DOI: https://doi.org/10.1016/j.jebo.2017.06.004.

Smick, D.M., (2009). O mundo é curvo: Perigos ocultos para a economia mundial. Rio de Janeiro: Best Seller.

Smith, J. D. (2016). US political corruption and firm financial policies. Journal of Financial Economics, 121(2), 350-367. DOI: https://doi.org/10.1016/j.jfineco.2015.08.021.

Shiller, R. J., (2012). Finanças para uma boa sociedade: Como o capitalismo financeiro pode contribuir para um mundo mais justo. Rio de Janeiro: Elsevier.

Shikida, C., Paiva, G. L., \& Junior, A. F. A. (2016). Análise de quebras estruturais na série do preço do boi gordo no Estado de São Paulo. Economia Aplicada, 20(2), 265-286.

Transparency International. (2012). Greece: The Cost of a Bribe. Disponível em:< https://www.transparency.org/ news/feature/greece the cost_of_a bribe>. Acesso em outubro/2019.

Transparency International. (2018). Disponível em: <https://www.transparency.org $>$. Acesso em dezembro/2018.

Vale, S. R. (2018). Macroeconomia e governança global: um casamento incompleto. Relações Internacionais no Mundo Atual, 1(24). 